been obtained in lying-in hospitals and maternities by the adoption of antiseptic measures in the elimination of septic processes are not as yet apparent in obstetric practice generally throughout the country. The natural inference is that no approach towards the general adoption of antiseptic measures has yet been roade. That this state of things exists is, on consideration, not a matter for surprise. For but a small proportion of obstetric practice is at present in the hands of those who have been educated in the use of antiseptics, and even of those who make a practice of following out aseptic and antiseptic principles but few do so in a really efficient manner. The reduction of puerperal mortality on any considerable scale is as yet a dream of the future, and it must take years before that dream will be fully realised. Already encouraging signs are apparent, in so far that the rise in the death-rate from puerperal fever which has taken place during the last decade in the provinces has not attained to corresponding proportions in London. In London and the larger towns, where antiseptic measures are naturally more freely adopted, improvement may first be looked for, and that the provinces will shortly follow suit may be confidently anticipated.

Weymouth-street, W.

\section{SOME CASES OF CEREBRAL DISEASE.}

\section{BY W. B. RANSOM, M.A. CAMB., M.D., M.R.C.P. LOND.,} PHYSICIAN TO THE GENERAL HOSPITAL, NOTTINGHAM.

THE following cases possess, I think, sufficient interest, from the difficulties of diagnosis or the peculiarities of symptoms, to be worth recording. Cases 1 and 2, 4 and 5, and 6 and 7 form natural clinical groups with diverse pathological processes.

CASE 1. Subaoute hydrocephalus. - A young married man aged twenty-three, without history of syphilis or tubercle, was suddenly, whilst at work, seized with pains in the head and back and general soreness. He went home and during the next week was half delirious and vomited frequently. Three weeks later he was admitted into hospital, having in the interval lost much flesh, and remained very weak and apathetic. On admission he was irritable, stupid, deficient in memory and occasionally exhibiting hallucinations. $\mathrm{He}$ complained of pain in the head and soreness all over the trunk and limbs. He was much emaciated, had a harsh dry skin and a temperature of $101.8^{\circ}$. Though there was general muscular wasting there was no actual paralysis, tremors or spasm, but he was only just strong enough to walk. There was no loss of general sensation, but slight general hyperæsthesia. The special senses were normal, except that he could not hear a watch tick further off than one inch. The tympanitic membranes were normal. His speech was somewhat drawling, the pupils were equal and reacted normally, the knee-jerks were normal, there was no ankle-clonus and the plantar reflexes were slight. There was slight double optic neuritis. There were no signs of visceral disease. The patient lived for a month, presenting a hectic temperature $\left(97^{\circ}\right.$ to $\left.103^{\circ}\right)$, whilst headache, vomiting and mental obscuration gradually grew worse. The only new phenomenon was the appearance of nystagmus a week after admission. $\mathrm{He}$ finally passed into stupor, became cyanosed, collapsed and died.

Necropsy. - There were no changes in the thoracic and abdominal viscera, except slight bronchitis. In the brain the convolutions were fattened; there were no lymph or tubercles anywhere. The lateral ventricles were distended with clear fluid to twice their normal size. The cortex was thinned. The foramina of Monro were much enlarged, as was also the fourth ventricle. On the velum interpositum there was a patch of firm fibrous thickening the size of a threepenny piece, apparently obstructing the veins of Galen. There was no clot in the veins. There was no tumour, nor were there any signs of inflammation of the choroid plexuses or of the brain substance, and there was no obvious thickening of the roof of the fourth ventricle. The spinal cord appeared soft in its upper half, but showed no marked change under the microscope.

An exact diagnosis in this case was not possible, and even the pathology remains obscure. The fibrous patch may have been the remains of a gummatous inflammation, but in the absence of further proof $I$ do not consider the diagnosis of syphilis tenable. Nor, after careful search, could any trace of tuberculous inflammation be found. It seems probable that the thickening of the velum interpositum may have antedated the acute onset of the clisease, which might have been brought on by a new insignificant obstruction to the orifices of the fourth ventricle. The continued fever in the absence of discoverable inflammation is noteworthy.

Case 2. Cerebral syphitis. - This case is of interest from the resemblance in symptoms and difference in result to the last. A man aged thirty-seven had secondary syphilis a year previously, but took mercury for a few months only. Three months ago he married. He seems to have become drowsy and inattentive to business shortly before marriage, but whilst on the honeymoon he was attacked with headache, vomiting and rapidly progressing muscular weakness, with marked mental detcrioration After his return home he was often delirious. When I saw him (September, 1892) he closely resembled the previous case. He was greatly emaciated, pale, trembling, and with a hesitating, indistinct speech. There was marked mental dulness, and he was sometimes delirious and sometimes semicomatose. He could neither read nor write, nor name objects correctly until told. He could just walk a few steps. There was no localised paralysis or spasm, and no anæsthesia or hyperæsthesia. The special senses, so far as they could be tested, did not seem markedly affected. There was double ankle-clonus. The sphincters were normal. There was no optic neuritis. The other systems were normal. The temperature curve, unfortunately, could not be obtained. The patient was given large doses of iodide of potassium and he improved steadily. A note taken in April, 1893, says: "He looks plump and healthy, can walk ten miles, do a little business and play a good game of draughts. He reads easily, but occasionally sees double. The handwriting is still a little jerky and there is slight-tremor of the hands. No optic neuritis ; knee-jerks normal ; no ankle-clonus. For the last few weeks he has had some headache, which till then had been absent."

In this case the etiology seems clear, though the site and form of the cerebral lesion are uncertain. Endarteritis is less probable at this "late secondary" stage of the disease than in the tertiary stage, and Case 1 shows how a small gummatous deposit may, by inducing hydrocephalus, cause the symptoms exhibited. I incline to think that this is more probable than the diagnosis of a diffuse syphilitic meningitis or a large gummatous tumour.

CASE 3. Right hemiplegia with complete right facial paralysis and loss of taste. - A boy aged ten was admitted into hospital for loss of power over the right side, which had come on gradually during the previous six months.' There was no history of syphilitic or tuberculous disease or of injury to the head. On admission he was found to be an intelligent, well-nourished boy, free from pain and sickness. Speech was rather indistinct, but there was no aphasia. The gait was somewhat ataxic, the feet were kept wide apart and there was a tendency on the part of the patient to fall forwards or to either side. The right arm and leg were very weak, though not completely paralysed. There was also slight weakness of the left leg. There was complete right facial paralysis, including the orbicularis oculi and corrugator supercilii. The paralysis was observed in both voluntary and emotional movements. The tongue deviated to the right, but showed no wasting. The palate was normal as well as the pupils. There was no ptosis, but there was slight internal strabismus in both eyes. The left eye could not move outwards beyond the middle line and the right eye only slightly further. There was slow nystagmus of the latter whilst looking forward. The sphincters were normal. Both knee-jerks were increased, there was ankleclonus in the right leg; the tendon reflexes were increased in the right arm, but the plantar and cremasteric reflexes were absent. There was slight double optic neuritis. Sensation to touch and pain was everywhere normal, as were also smell and vision. He heard a watch tick four inches from the right ear and two inches from the left. 'The tympanic membranes appeared to be normal. There was complete loss of taste on the right side of the tongue, not only on the anterior two-thirds but on the posterior third. Tactile sensibility on the tongue and other areas of the fifth nerve was quite intact. The patient lived for ten weeks after admission. After three weeks he began to have headache and vomiting, which recurred at intervals until the end. The weakness and incoördination of the legs increased, so that after a month he could not walk and the ankle-clonus became double. The condition of the arms and facial muscles remained uncbanged, but shortly before death it was thought that the right half of the tongue was slightly 
wasted. The loss of taste persisted and a week before death tactile sensibility appeared to be very slightly dulled on the right side of the tongue near the tip, but there was no general anæsthesia in the area of the fifth nerve. Hearing gradually diminished in the right ear till he could only hear through the bone. There were also one or two attacks of severe pain in this ear. The sixth nerves remained paralysed and he became unable voluntarily to move the right eye to the left, though it still converged readily on accommodation. The optic neuritis increased slightly. The temperature remained normal or subnormal throughout. Death occurred unexpectedly, respiration suddenly ceasing, whilst the pulse remained regular and was felt for five minutes after the last voluntary respiratory movement.

Necropsy.-Beyond slight flattening of the convolutions the cerebral hemispheres showed no change. When the brain was removed the whole of the pons appeared hypertrophied and the right half of its lower surface was covered by a lobulated mass, which spread into the right lobe of the cerebellum. Both sixth nerves and the right fourth nerve were flattened and translucent. The right seventh and eighth nerves were red, swollen and cedematous. No change was found in the fifth nerves. On section the substance of the pons appeared nearly normal to the naked eye, but there was a patch of hæmorrhagic softening in the adjacent part of the right hemisphere of the cerebellum. The microscope showed an infiltrating glioma, spreading through the whole breadth of the pons, but more bulky in its right half, where it grew up the middle peduncle into the cerebellum. The right chorda tympani was dissected out in the middle ear and on microscopical examination showed marked signs of degeneration.

Although in life the symptoms pointed to a cerebellar tumour, it was difficult to explain the association of right hemiplegia with paralysis of the upper part of the facial nerve and the loss of taste on the assumption of a single focus of disease. The only moderate deafness and the absence of much pain in the right ear contra-indicated the diagnosis of a malignant growth invading the right petrous bone and the canals of the seventh and eighth nerves, especially as it appeared that the main mass of the growth pressed upon the left pyramidal tract and ought therefore to be in the left half of the cerebellum. The necropsy showed the extraordinary combination of symptoms which may be produced by the infiltration of a glioma, or what is not inaptly termed the process of gliomatosis. Even when examining by the microscope one feels doubtful whether a given part is diseased or natural, and it appears from this case that the interference with function is not measured by the visible magnitude of the growth; for, though it was the left pyramidal tract whose function was most disturbed, it was in the right half of the pons that the tumour was most distinct and so large as to press on the right fourth nerves and to injure the seventh and eighth. Such a tumour creeps insidiously between the nerve elements, and may in one part, though its bulk be large, do them but little harm, whilst in another, though less voluminous, it damages the activity of both nerve fibres and cells. I did not, unfortunately, ascertain whether the redness and swelling of the seventh and eighth nerves were inflammatory or due to is spread of the glioma down them, as these nerves broke off in the hardening process. The latter view is the more probable, as the pressure of the tumour on other nerves (sixth and fourth) causes them to be pale and flat, instead of red and swollen. The degeneration of the chorda tympani (demonstrated in osmic acid and carmine preparations) is of interest in regard to the course of the fibres of taste. The case would at first sight seem to point to the chorda degeneration being due to disease of the root of the seventh nerve, but further consideration shows that this is not a necessary conclusion; for the swelling of the facial nerve extended so far into the bony canal that it undoubtedly pressed upon the chorda tympani fibres there, and it is therefore probable that their injury was due to this mechanical pressure and not to a degeneration descending from the seventh root. There was no evidence, either clinical or anatomical, of disease of the fifth nerve, and no chang'e was visible to the naked eve in the roots of the glosso-pharyngeal or vagus nerves, which were behind the mass of the tumour. The loss of taste in this case was comparable to that occurring in neuritis of the seventh nerve in the Fallopian aqueduct, and the facts do not advance knowledge of the course of the taste fibres on the central side of the junction of the chorda tympani and the facial nerves. It may be noted that, so far as could be ascertained, taste was lost on the posterior third as well as on the anterior two-thirds of the tongue. This point is, however, difficult to test with accuracy in children. I may also mention here two surgical experiments in which, owing to the kindness of my colleague Mr. Chicken, I was able to further investigate the sense of taste. In the one case he had excised a portion of the right lingual nerve and in the other a piece of the right supra-maxillary, with Meckel's ganglion, both for severe neuralgia In the former case the right lingual nerve was reached through the sigmoid notch of the inferior maxilla and a piece excised two years ago. The patient now has complete anæsthesia and loss of taste on the right side of the tongue in its anterior two-thirds. On the posterior third taste is normal and tactile sensibility is nearly as acute as on the other side of the tongue. Touch and taste are also normal on the palate. This observation supports the numerous experiments which have been beld to show that the taste fibres in the chorda supply only the anterior two-thirds of the tongue. In the second case Meckel's ganglion and a portion of the infraorbital nerve were removed a year ago. For a time tactile sensation was diminished on the cheek, but it has now returned nearly to normal throughout the area of distribution of the supra-maxillary division of the fifth nerve, though the pain has not returned. At no time has taste been affected, as it would have been if the taste fibres reached the brain by way of the Vidian nerve and the second division of the fifth nerve.

CASE 4. Tumour of corpus striatum. - This was the case of a man aged forty-seven, an intelligent, temperate artisan, without history of syphilis or tubercle. Ten weeks before admission into hospital he began to be apathetic, drowsy and to have some headache. $\mathrm{He}$ is said also to have "talked queerly." During the last three weeks he had slept a great deal, had had some delusions, failure of memory and loss of interest in his surroundings. For a fortnight he had been unable to stand without support, and his wife thought that at first his left leg was weak. There had been considerable frontal headache and occasional vomiting. The day after admission he was very drowsy, only answering questions after vigorous rousing. The right hand was mostly kept on the forehead, which he said was painful. There was some general cephalic tenderness. He occasionally made clutches with either hand at space. He could not feed himself and could not stand alone, but there was no actual paralysis of the eyes, face or limbs and no anæsthesia. There was very slight rigidity of the left biceps, but no difference between the legs. The knee-jerks were diminished, there was no ankle-clonus and the plantar reflexes were present. He sometimes micturated in bed. The pupils were of medium size, equal and they reacted normally. There was slight double optic neuritis. The temperature was subnormal. There was no sign of disease of the thoracic or abdominal viscera. The arine was normal. The patient lived for ten days after admission, the drowsy condition gradually deepening into coma. The slight rigidity of the left arm was not again observed, and no other spasm or paresis developed. The temperature remained between $97^{\circ}$ and $98^{\circ}$ till the day of death, when it rose to $100 \cdot 6^{\circ}$

Necropsy. - The frontal convolutions were flattened. There was slight excess of fluid in the lateral ventricles. The fornix was soft and almost diffluent. The right caudate nucleus was practically replaced by a growth but little larger than the left caudate nucleus. The tumour was in parts soft, gelatinous and reddish; in parts barder and white. On microscopical examination it proved to be a myxo-gliosarcoma. The rest of the brain was normal. Except for some old pericardial adhesions there was no disease of the thoracic and abdominal organs.

The diagnosis of intra-cranial tumour was in this case easy, though its localisation was not. Beyond the slight weakness of the left leg, asserted to have been present by his wife before he came under my notice, and the doubtful temporary rigidity of the left arm, there was no indication of pressure on the internal capsule. In view of recent physiological research on the corpus striatum as a thermo-genetic centre, the absence of fever in this case is of interest, for the development of the symptoms was fairly rapid, so that signs of interference with the functions of this organ might have been expected. It would seem that only sudden lesions, such as those caused by the experimenter or such as hæmorrhage, are capable of producing an obvious effect on the regulation of heat. Probably a great part of the caudate nucleus had been slowly destroyed by the more chronic parts of the tumour, and the final rather rapid obliteration of the functions of the brain 
was due to increase in the general intra-cranial pressure caused by extension of its sarcomatous portion.

CASE.5. Lead encephalopathy. - This case at first showed considerable clinical resemblance to the last, but in its cause and its result differed altogether. A young man aged seventeen, a carriage painter, was sent into hospital for cerebral tumour. His only previous illnesses were measles and "gastric fever." For three weeks before admission he suffered from headache, vomiting, dimness of vision, and pain in the abdomen. He repeatedly tried to work, but always had to give up after three days, becoming somewhat better again after a few days' rest. On inquiry it was found that the vomiting had been chiefly after food, that the bowels were confined, and that the pain in the abdomen was colicky in character. There was no history or sign of syphilis. Four days after admission the patient was found to be a well-nourished but rather anæmic lad. He was lethargic and complained of continuous frontal headache, which at times became intense. There was no ocular, facial or limb paralysis, and there was no spasm and no anæsthesia. The knee-jerks were normal, as were also the plantar reflexes. The pupils were normal. There was well-marked double optic neuritis and a large retinal hæmorrhage in the left eye. He had some nausea and occasional vomiting. The bowels were then open, and the urine was free from albumen or sugar, of sp. gr. 1012 to 1016, and amounting daily to about $30 \mathrm{oz}$. The pulse was soft and regular. The heart's apex was displaced two inches to the left, and a soft systolic murmur was audible over it. The tongue was moist, furred and tremulous. There was a slight blue line on the gums. For treatment the bowels were kept open and iodide and bromide of potassium were given. with an occasional dose of antipyrin for the exacerbations of headache. A fortnight later he was no better, and then tincture of digitalis was added to his mixture. Within a week from this marked improvement was observed and all his symptoms had left him five weeks after admission. The vision improved and the optic neuritis became perceptibly less, but some yellow striæ developed round each macula lntea. During the last ten days the daily output of urine increased to fifty ounces, but its specific gravity decreased to 1006 to 1010, and the urea was found to be but 1 per cent. Four times during his five weeks in hospital a trace of albumen appeared in the urine. The blue line on the gums diminished, but had not quite disappeared when he was discharged.

Lead encephalopathy, tolerably frequent amongst the white-lead workers of Newcastle, is very rare in Nottinghamshire, though the more usual phenomena of plumbism, such as colic and wrist-drop, are not uncommon. This patient had been engaged for two years in "fine painting," during which time he remained healthy, but for three months before admission had taken to coarse work, in which much red lead was used, and had suffered in consequence an acute intoxication. In him, as in the girl workers in white lead mentioned by Dr. Oliver, the sudden introduction of lead into the system caused serious cerebral disturbance without any of the peripheral nerve degeneration usual in chronic plumbism. There was evidence also in the low specific gravity of the urine and the occasional albuminuria that the kidneys were affected by the poison, and it is probable that the condition of granular kidney will supervene. The fact that improvement did not commence until the iodide of potassium was supplemented by digitalis may be associated with the fact that there was cardiac dilatation without hypertrophy, which, again, was probably connected with the subacute renal degeneration. It is also of interest in connexion with Dr. Dixon Mann's recent statement that iodides do not increase the elimination of lead from the system. In a case of wrist-drop from chronic plumbism recently under my care, which improved under iodides, I may mention that there was distinct anæsthesia in the area of distribution of the right radial and part of the right ulnar nerves, thus supporting the assertion of Dr. Judson Bury that the neuritic affection is not always confined to the motor nerves.

The two following cases illustrate the difficulties of diagnosis in typhoid fever.

CASE 6. Tuberculous meningitis.-A girl aged sixteen was sent into hospital for typhoid fever. She had been ailing for a fortnight, and was in bed for five days with fever, headache, and slight delirium, but no diarrhoea. On admission she was a well-nourished girl, lay on her back in a drowsy state, objected to being moved, and showed that her limbs were tender. The temperature was $101^{\circ}$, the pulse 104 and the respiration 20. The tongue was furred, the abdomen was full, there were no rose spots and the spleen could not be folt. $A$ few rîles were heard scattered over the lungs. The heart was normal. The right pupil was larger than the left, but both reacted normally. There was no paralysis of the external ocular muscles, face, tongue or linbs. The knee-jerks were normal. There was no optic neuritis. The hearing was normal, as was also the urine. The only symptoms pointing to brain disease rather than typhoid fever were the inequality of the pupils and the fact that the patient was rather more irritable than is usual in fever patients; but the former might have been natural to her, and the latter might be attributed to the nervousness of a girl above the usual class of hospital patients on first being brought amongst strangers. The normal condition of the kneejerks and the absence of optic neuritis opposed the idea of cerebral mischief. For five days there was no change in the symptoms and the diagnosis remained doubtful; but on the sixth day the drowsiness increased, the knee-jerks disappeared and there was slight retraction of the head. The optic discs were still normal. On the seventh day after admission the patient passed into a state of stupor and on the eighth day, whilst still comatose, she died. The temperature had varied from $99^{\circ}$ to $102^{\circ}$, but was subnormal just before death.

Necrop:y. - There were tubercles in the pia mater all over the base of the brain, along the Sylvian fissures and on the cerebellar arteries. The cerebral ventricles were somewhat distended. Miliary tubercles studded tbe lungs and spleen, and there were a few in the kidneys and under the capsule of the liver. There was no inflammation or ulceration of the bowels.

A positive diagnosis during the first few days was, I think, impossible, although we inclined to the view of meningitis. It should be noted that the knee-jerks failed as a test, since they were retained till late in the disease, nor was headache ever a marked symptom. The absence of diarrhœa and the non-discovery of rose-spots and enlarged spleen are so common in typhoid tever-at least, in its early stages-that much importance conld not be attached to these facts.

CASE 7. Typhoid fever and cerebellar tumour.-A young woman aged seventeen was sent into hospital with the diagnosis of typhoid fever from a house where four children had that disease. She had been in bed for sixteen days, suffering from headache, shiverings, cough and slight diarrhœa. Four days after admission (Feb. 13th) she presented the usual appearance of a typhoid fever ratient at the end of the second week. She rambled somewbat in her talk; the abdomen was slightly distended, the spleen could be felt, but no rose-spots were visible. There were rhonchi in both lungs and a systolic bruit was heard at the heart's apex. The urine was normal. There was some stiffness of the neck. The pupils were equal, large and reacted sluggishly. There was no ptosis. There was some weakness of both external recti and of the left internal rectus. There was double optic neuritis. There was no facial, lingnal or limb paralysis. The knee jerks were absent. The sphincters were normal. There was no sign of ear disease. The temperature was $1024^{\circ}$, the pulse 104 , and the respira. tion 26. On $\mathrm{reb}$. 21 st the patient seemed better. The mental condition was fairly good, there was no delirium and no headache. The ocular paresis was less marked. The temperature varied from $99^{\circ}$ to $101^{\circ}$. There was no diarrhoea. On the 25 th she said she was better. The knee jerks had returned, the plantar reflexes were exag. gerated, and the optic neuritis had increased. Temperatue, $99^{\circ}$ to $1034^{\circ}$. On the 27 th there was intestinal hæmorrhage, about three quarters of a pint of blood being passed. On the 28th the breathing became sighing and the patient passed into a state of stupor. Purpuric patches appeared on the arms and legs. Both arms were rigid and flexed. She could only move the shoulder of the right arm and the wrist of the left. Both legs were rigid and she could only move the right one slightly. Knee-jerk was absent on the right, but present on the left side. The plantar reflexes were still exaggerated. She was sensitive to a pinch on any part of the body. The pupils were contracted and there was nystagmus of the right eye. The abdomen was retracted. The temperature was $1004^{\circ}$, the pulse 150 , and respiration 16 . On the 29th the patient was comatose and the limbs were flaccid. There were numerous petechir on the limbs and under the conjunctiva. Death supervened. The temperature was $98^{\circ}$ a few hours before death.

Necropsy. - There were typical typhoid ulcers in the ileum, as well as numerous small hæmorrhages under the visceral an $l$ parietal peritoneum, in the lungs and kidneys, in the pricranium, choroid plexuses and septum lucidum. In the 
right lobe of the cerebellum there was a tumour, an inch and a half in diameter, apparently growing from the pia mater and pressing deeply into the cerebellum and somewhat into the pons, from both of which it readily separated. There was much hæmorrhage in the deepest part of the tumour. There was no free blood in the ventricles of the brain or in the cranial cavity. On microscopical examination the tumour proved to be a fibroma.

The diagnosis was in this case peculiarly difficult, there being strong evidence both of typhoid fever and of cerebral disease, and the coexistence of the two being rare. So far as we could learn the tumour had produced no symptoms before the attack of fever, although it must have been present some time previously. Very soon after admission the strabismus, optic neuritis and loss of knee-jerks showed the existence of organic disease of the brain, but for a fortnight this did not appear to have an unfavourable influence on the disease. Then a purpuric condition seems to have set in, causing hæmorrhage into the bowel, subcutaneous tissue, lungs and kidneys, and probably the hæmorrhage into the cerebellar tumour. This last occurrence may be considered as the immediate cause of death. Extensive purpura must be rare in typhoid ferer, and the question may be asked whether the tumour in any way led to its development.

Nottingham.

\section{TREATMENT OF WOUNDS WITH IZAL.}

By WM. BRUCE CLARKE, M.B. OxoN., F.R.C.S. ENG., ASSISTANT SURGEON TO ST. BARTHOLOMEW'S HOSPITAL ETC.

IN November of last year Dr. Klein mentioned to me that he had been making some experiments with a new substance called "izal," " which exhibited very remarkable disinfectant properties, was non-irritant and had at the same time nopoisonous influence on any of the higher animals even in extremely concentrated solutions. After carefully reading Dr. Klein's report on the subject and the account of his experiments, and after conferring with him upon the subject, I determined to test its efficiency and its applicability as a surgical disinfectant, and now, after some six months' trial, I am anxious to record the result of my investigations, but before doing so it may be advisable to recall Dr. Klein's statements :-

"Now I have to add a simple statement with reference to all species mentioned as $5,6,7,8,9,10,11,12,13,14,15$, $16,17,18,19,20,21$ and $25,{ }^{2}$ for in all these instances the disinfectant used in the strength of 1 in 200 completely destroys in five minutes the vitality of the microbes; no living cultures can be produced with them. The experiments were made at first also in this instance with the disinfectant of the strength of 1 in 100 , exposing the microbes in it for five, ten and fifteen minates, and also to 1 in 200, exposure fifteen minutes; but it need hardly be said that all vitality was destroyed, as already 1 in 200 for five minutes is a complete disinfectant. (It may not be unnecessary to state here that the same cultivations of bacillus diphtheriæ and bacillus of typhoid used in this series of experiments-namely, exposure in the disinfectant of the strength of 1 in 200-were used, and with exactly the same methodsalso, for comparative experiments of disinfection with carbolic acid. Crystals of absolute phenol were dissolved in water so as to form a solution of the strength of 1 in 200 ; the microbes were then introduced into this solution and exposed to its action for various periods. As a result it was found that an exposure for two hours in 0.5 per cent, carbolic acid-

1 The substance was at that time known by the term of the "Thorncliffe disinfectant." It has since been renamed "zzal." It is a by.product cliffe disinfectant." It has since been renamed " "zal." It is a by.product obtained by Messrs. Newton, Chambers and co. of the Thorncliffe Ironwave been gocd enough to place some at my disposal for experimental have been
purposes. ing: (1) Spores of bacillus subtilis (hay bacillus); (2) spores of bacillus mesentericus (a common putrefactipe microbe); (3) spores of bacillus anthracis; (4) bacillus subtilis, non spore bearing forms; (5) bacillus mesentericus, non-spore bearing forms; (6) bacillus anthracis of the blood of an animal dead of virulent anthrax ; (7) bacillus of diphtheri 1 (8) bacillus of typhoid fever; (9) bacillus of glanders; (10) bacillus of septicamia (mouse); (11) bacillus of fowl cholera; (12) bacillus of fowl enteritis; (13) bacillus of pneumonia; (14) bacillus of gronse disease (15) bacillus of swine fever; (16) hacillus of swine erysipelas; (17) bacillus prodigiosus (pink), non-pathogenic; (18) spirillum of Finkler; (19) spirillum of cholera (cholera bacillus); (20) staphylococcus pyogenes aureus (21) staphylococcus pyogenes albus; (22) streptococcus pyogenes (23) streptococcus of erysipelas; 24) streptococcus of scarlatina (25) proteus vulgaris (the common microbe of putrefaction). i.e., 1 in 200-does not in the least injure the bacilli ; good and typical growth can be established with them after the exposure. With regard to species 22,23 , and 24 (streptococci), these I knew from previous experiments with other disinfectants to be less resistant than the staphylococci and bacilli. I have therefore subjected these three species to the disinfectant in weaker strength-namely, 1 in 300-and found that an exposure for five minutes in this mixture is fatal to these microbes: streptococcus pyogenes, streptococcus of erysipelas, and streptococcus of scarlatina. From these series it follows that an exposure for five minutes in the strength of 1 in 200 ( 1 in 200 would mean one ounce of the disinfectant emulsion distributed in ten pints of water) completely destroys the vitality of the microbes of diphtheria, typhoid fever, fowl cholera, swine fever, glanders, cholera, of suppuration, of erysipelas, scarlatina, and other non-sporing pathogenic and non-pathogenic species."

In addition to these experiments on the destruction of microbes Dr. Klein made some researches as to the solutions that should be used with a view to inbibiting their growth, and states

"The result of all experiments on inhibition may be briefly summarised for all the twenty-five species tested-namely, (1) Spores do not germinate in medicated gelatine or broth if the amount of disinfectant added is 0.1 per cent. ; (2) no micrococcus (staphylococci and streptococci) is capable of growing in medicated gelatine or broth of the strength of 0.1 per cent. medication; (3) all non-sporing bacilli and spirilla fail to grow in medicated gelatine or broth of the strength of 0.1 per cent. medication; (4) excepted from this are the bacillus prodigiosus and the bacillus of typhoid-the bacillus prodigiosus shows growth on gelatine medicated 0.1 per cent. the growth is retarded, and always starts from the superficial layer of the medium; it proceeds considerably slower than on normal gelatine."

These exceptions obviously do not concern one from a surgical point of view, inasmuch as bacillus prodigiosus is not a pathogenic organism at all, and the bacillus of typhoid fever does not enter wounds. It follows from these experiments that for destructive purposes a solution of 1 in 200 of the fluid is all that is required, whilst for inhibition purposes 1 in 1000 is quite sufficient. My task then was limited to determining how far solutions of this strength are available for surgical purposes and to ascertaining whether they possessed any advantages over the antisentic solutions ${ }^{3}$ in ordinary use, such as carbolic acid, corrosive sublimate \&c.

The cases in which $I$ have used it have been selected by me because their behaviour under the more commonly used antiseptics is so well known that it may be regarded almost in the light of a control experiment as regards this new disinfectant. The samples of the fluid which were submitted to me were diluted down to a strength of 1 in 50 , and kept in bottles ready for use, the subsequent dilution to 1 in 200 being effected at the actual time of operation. The sponges which were to be used at the operation were cleansed, as is my ordinary custom, in soda-and-water and then rinsed several times in fresh water until they no longer caused any turbidity in it. After this they were placed in a jar of izal ( 1 in 200) and kept there for at least twenty-four hours before use. The gauze which was employed as a dressing was the ordinary gauze of commerce boiled for twenty minutes in a saucepan over the fire and then soaked for twentyfour hours at least in a solution of izal (1 in 200) like the sponges. The gauze was wrung out just before use and placed in a moist condition over the wound without the intervention of any protective or other similar substance between it and the wound. A sufficient number of folds were used to allow for any possible soakage that could take place and the outside was then covered by any substance--e.g., cotton wool \&c.-which happened to be at hand in order to prevent the bedclothes from being wetted. The instruments were

3 I have used the word "solution" here and in all subsequent instances in reference to this fluid "izal," but to be strictly correct the word "emulsion" should be employed, and herein lies, in my opinion, a orce the chief advantage and disadvantage of the fiud in question. Being an emulsion and not a clear solution it is not so easy to see one's instruments when they are covered by it. At the same time, it must b borne in mind that the very fact that the fluid is so singularly unable to form chemical compounds with living animal tissues is in all probabilit highly advantageous from the germicidal point of view. Unlike coriosive sublimate, which speedily loses its disinfectant power by com bining with the albuminous substances of the body, izal retains this power in such a remarkable degree because it will not combine with anything. It is also at the ordinary temperature of the air practically non-volatile, as may be easily tested by placing a few drops on a piece of cotton wool and leaving it for several months in an exposed position. 